\title{
The Antihypertensive Effect of ASA Lasts Less than 24 Hours?
}

\author{
I. Suomela, J. Varis*, I. Kantola \\ Division of Medicine, Turku University Hospital, Turku, Finland \\ Email: ${ }^{*}$ juha.varis@tyks.fi
}

Received 26 January 2015; accepted 12 March 2015; published 19 March 2015

Copyright (C) 2015 by authors and Scientific Research Publishing Inc.

This work is licensed under the Creative Commons Attribution International License (CC BY). http://creativecommons.org/licenses/by/4.0/

(c) $\underset{\mathrm{EY}}{\mathrm{i}}$ Open Access

\begin{abstract}
Objectives: Some studies suggest that acetylsalicylic acid (ASA) administered in the evening may have a lowering effect on the blood pressure. The purpose of this study was to measure the effect of the time of ASA administration on blood pressure, markers of arterial stiffness as well as various plasma parameters. Methods and Results: The study included 34 patients $(20$ men and 14 women) ranging in age from 47 to 82 years. Low-dose ASA (50 - $250 \mathrm{mg} / \mathrm{day})$ was used by all the patients, first in the morning and later in the evening for three months. Home, office and ambulatory blood pressure and pulse wave velocity (PWV) were measured. Compared with the morning administration of ASA, significantly lower office systolic blood pressure $(p=0.017)$ and significantly higher carotid-femoral PWV $(p=0.010)$ as well as plasma triglycerides $(p=0.002)$ were found after the evening administration. There were no statistically significant changes in the average ambulatory blood pressure values, carotid-radial PWV or other parameters measured. However, the time of ASA administration seemed to affect the circadian variation in blood pressure. Conclusions: The results suggest that ASA administration may lower blood pressure, but for less than 24 hours. Our results should be verified by larger, randomized studies.
\end{abstract}

\section{Keywords}

Acetylsalicylic Acid, Blood Pressure, Arterial Stiffness, Pulse Wave Velocity, Stiffness Index, Reflection Index

\section{Introduction}

Acetylsalicylic acid (ASA) has been used in the prevention of cardiovascular events [1]. Patients with hypertension use ASA in addition to blood pressure medication, typically administered in the morning. Hermida et al. has

${ }^{*}$ Corresponding author. 
studied the effects of the evening administration of ASA versus the morning administration in pregnant women [2], healthy subjects [3], patients with pre-hypertension [4] and patients with untreated hypertension [5]-[7]. They found ASA administered in the evening, but not in the morning, to have an antihypertensive effect. In contrast, Dimitrov et al. [8] did not find the evening administration of ASA to have a beneficial effect on ambulatory blood pressure (BP) compared with the morning administration. Parallel results are recently published by Bonten et al. (2015) who used $100 \mathrm{mg}$ ASA and Lafeber et al. whose polypill contained $75 \mathrm{mg}$ ASA (2014) [9] [10]. Consequently, the BP lowering effect of bed-time ASA as a whole is still unclear and warrants more studying.

Arterial pulse wave velocity (PWV) is a marker of elastic artery stiffening and an independent predictor of cardiovascular mortality [9]. In addition to PWV, arterial stiffness can also be measured by determining the stiffness index (SI) derived from the digital volume pulse (DVP) [10]. This has been shown to be a reproducible technique with a comparable sensitivity and specificity to the PWV method in the identification of patients with latent CVD [11]-[13]. The reflection index (RI), describing the size of the reflection wave, is also derived from DVP.

In the present study, the effects of the time of ASA administration was studied using well controlled Finnish hypertensive patients. Office and ambulatory blood pressure, carotid-femoral and carotid-radial PWV, RI and SI as well as on various plasma parameters were studied.

\section{Materials and Methods}

\subsection{Subjects}

The study population was recruited among successive and adequately drug-treated hypertensive patients in the outpatient clinic of the Department of Medicine of the Turku University Hospital, Turku, Finland.

34 patients (20 male and 14 female) aged 47 - 82 years (mean $64.9 \pm 7.6$ years) were studied. Low-dose ASA ( 50 - $250 \mathrm{mg} /$ day) was used by all the patients, and they had been on regular antihypertensive drug therapy for at least one year. Patients with any other regular drug therapy affecting the cardiovascular system were excluded. The history of the drug use was based on self-reporting on the day of the office BP measurement and, whenever possible, verified from the local patient files. The patient characteristics are presented in Table 1.

\begin{tabular}{|c|c|}
\hline Variable & \\
\hline Patients $(n)$ & 34 \\
\hline Sex, \% men & 58.8 \\
\hline Age range $(y)$ & $47-82$ \\
\hline Age $(y)$ & $64.9 \pm 7.6$ \\
\hline Height (cm) & $174.2 \pm 10.6$ \\
\hline Weight (kg) & $92.6 \pm 18.5$ \\
\hline $\operatorname{BMI}\left(\mathrm{kg} / \mathrm{m}^{2}\right)$ & $30.4 \pm 4.7$ \\
\hline Waist (cm) & $107.2 \pm 14.3$ \\
\hline Monotherapy ( $n$ ) & $6(17.7 \%)$ \\
\hline ACE inhibitor $(n)$ & 1 \\
\hline ATR blocker $(n)$ & 2 \\
\hline$\beta$-blocker $(n)$ & 1 \\
\hline Calcium antagonist (n) & 0 \\
\hline Central acting agents $(n)$ & 0 \\
\hline Diuretic $(n)$ & 1 \\
\hline Combination therapy & 28 (82.3\%) \\
\hline
\end{tabular}

${ }^{a}$ Results expressed as mean $\pm \mathrm{SD}$; $n$, number of participants; ACE, angiotensin converting enzyme; ATR, angiotensin receptor. 
The study was conducted at the Turku University Hospital between May 2010 and November 2010. The study protocol was evaluated and approved by the ethics committee of the Hospital District of Southwest Finland. Prior to the study, the patients were informed about the research and they had an opportunity to ask questions. Patients were asked to sign a written consent and to fill in a 15d questionnaire and a SF-36 health survey. The results were not utilized in this study.

All the patients had been receiving ASA (50 - 250 mg per day) on awakening for at least one year. In the beginning of the study the measurements were performed with the patients using the morning dose of ASA. Thereafter the patients were assigned to receive the same dosage of ASA (50 - $250 \mathrm{mg}$ per day) at bedtime for 3 months. At the end of this period, the measurements were repeated. No changes to other medicines or their dosages were made.

Compliance was measured on the basis of a personal interview with each patient. The patients performed duplicate measurements of the systolic blood pressure (SBP) and diastolic blood pressure (DBP) as well as heart rate for 1 week, both on awakening and at bedtime [14]. After this, before the first clinic visit, blood samples from antecubital vein were obtained between 8 am and 9 am after nocturnal fasting. Plasma total cholesterol, HDL-cholesterol, LDL-cholesterol, triglycerides, glucose and other common laboratory chemistry values were measured by standard methods. ECG was also taken by the University hospital laboratory personnel. During the clinic visit PWV, RI and SI were measured. On the same day, 24-hour ABPM was initiated. Office BP was measured twice with a 2-min interval after at least a 10-min rest using a calibrated digital sphygmomanometer with the appropriate cuff sizes. The measurements were always obtained by the same investigator. Details of the measurements are explained later.

After the measurements, patients were assigned to receive the same dosage of ASA (50 - $250 \mathrm{mg}$ per day) at bedtime for 3 months. At the end of this period, the measurements were repeated. No changes to other medicines or their dosages were made.

\subsection{Home BP Assessment}

Patients received written instructions and individual guidance on how to measure the BP correctly. They were instructed to measure seated BP twice, approximately at 2-min intervals every morning between 6 am and 9 am and every evening between $6 \mathrm{pm}$ and $9 \mathrm{pm}$ on seven consecutive days. Home BP was determined as the mean of approximately 14 duplicate measurements (28 measurements). The number of measurements varied between 8 and 28.

\subsection{4-Hour ABPM}

The 24-hour ABPM was initiated during the first clinic visit. The device used was Space Labs 90207. The SBP, DBP, pulse pressure, mean arterial pressure (MAP), and heart rate (HR) of each patient were automatically measured every 20 minutes during the day $(7 \mathrm{am}-10 \mathrm{pm})$ and every 30 minutes during the night (10 pm - $7 \mathrm{am})$. The ABPM was conducted on a normal weekday and the patients were instructed to follow their normal daily routine. The patients were instructed how to turn off the measuring device after 24 hours. The machines were returned by the patients on the following day and data was obtained from them by the investigator.

\subsection{Measurement of PWV, RI, and SI}

Carotid-femoral PWV and carotid-radial PWV were measured by a doppler probe (Pulse Trace PWV, Micro Medical Ltd). Proximal (carotid) and distal (radial and femoral) pulses were obtained and the PWV values were calculated from the transit time between the proximal and distal arterial sites, determined in relation to the Rwave of the ECG. The distance (in $\mathrm{cm}$ ) for calculating the carotid-femoral PWV was measured from the proximal end of sternum to the femoral measuring point using a measuring tape. The distance for calculating carotidradial PWV was measured by adding the distance between the proximal end of sternum and shoulder and the distance between shoulder and the radial measuring point at the wrist.

DVP was assessed with a photoplethysmograph (Pulse Trace PWV, Micro Medical Ltd.) as a function of time. DVP was measured from the index finger for 10 seconds after which the average values of RI and SI were calculated based on the systolic and the diastolic peaks of the DVP graph [15]. 


\subsection{Statistical Analyses}

All analyses were performed with SPSS software (SPSS Inc., Chicago, IL), version 19.0. Comparisons between the measurements after the different times of ASA administration were calculated with paired $t$-test when the data were normally distributed and with Wilcoxon Signed-rank test when they were not. The normal distribution of the continuous variables was confirmed using Shapiro-Wilks' test of normality. The significance of correlation between different parameters was determined by Pearson's (continuous variables) and Spearman's rho (categorial variables) correlation analysis. Two-sided tests and significance levels were used throughout.

\section{Results}

Patient details are presented in Table 1. As shown in Table 1, in average, the patients selected for the study were shown to be obese (BMI 30.4). Antihypertensive monotherapy was used by $17.7 \%$ and $82.3 \%$ of the patients received combination therapy. Out of the original 34 patients, 33 completed the ABP measurements.

Compared with the morning administration of ASA, a significant decrease in office SBP $(p=0.017)$ and a significant increase in carotid-femoral PWV $(p=0.010)$ as well as in plasma triglycerides $(p=0.002)$ were found after the evening administration of ASA (Table 2). These differences were found in both males and females (data not shown).

Table 2. Changes in measured parameters in subjects $(\mathrm{N}=34)$ completing the study as per protocol ${ }^{a}$.

\begin{tabular}{ccc}
\hline Measure & Morning ASA & Evening ASA \\
\hline Systolic office BP (mmHg) & $137.9 \pm 15.3^{*}$ & $132.3 \pm 17.3^{*}$ \\
Diastolic office BP (mmHg) & $82.4 \pm 8.1$ & $81.1 \pm 9.1$ \\
Heart rate (bpm) & $65.8 \pm 11.7$ & $68.3 \pm 10.3$ \\
PWV (C-R) (m/s) & $12.7 \pm 3.5$ & $12.3 \pm 1.8$ \\
PWV (C-F) (m/s) & $10.8 \pm 2.7^{*}$ & $11.4 \pm 2.2^{*}$ \\
RI KA /\% & $72.7 \pm 11.2$ & $70.8 \pm 12.5$ \\
SI & $10.7 \pm 2.1$ & $11.2 \pm 5.4$ \\
Total cholesterol (mmol/L) & $4.4 \pm 0.9$ & $4.5 \pm 0.9$ \\
HDL-c (mmol/L) & $1.5 \pm 0.5$ & $1.4 \pm 0.5$ \\
LDL-c (mmol/L) & $2.2 \pm 0.7$ & $2.2 \pm 0.7$ \\
Triglycerides (mmol/L) & $1.4 \pm 0.7^{* *}$ & $1.8 \pm 1.1^{* *}$ \\
Glucose (mmol/L) & $7.3 \pm 1.9$ & $7.4 \pm 2.2$ \\
GHbA1c (mmol/ml) & $52.6 \pm 11.1$ & $51.7 \pm 12.3$ \\
Creatinine (umol/L) & $81.1 \pm 16.8$ & $87.9 \pm 38.3$ \\
PQ time (ms) & $176.1 \pm 28.8$ & $173.0 \pm 29.6$ \\
QRS width (ms) & $97.7 \pm 16.9$ & $95.8 \pm 15.1$ \\
QTc (ms) & $431.1 \pm 23.6$ & $431.8 \pm 23.1$ \\
Syst BP morning, home ${ }^{d}$ & $136.7 \pm 18.4$ & $135.8 \pm 16,9$ \\
Diast BP morning, home & $81.7 \pm 9.9$ & $81.7 \pm 9.4$ \\
Syst BP evening, home & $135.2 \pm 16.6$ & $136.5 \pm 15.4$ \\
Diast BP evening, home ${ }^{e}$ & $80.1 \pm 10.6$ & $81.2 \pm 9.3$ \\
\hline
\end{tabular}

${ }^{a}$ Results expressed as mean $\pm \mathrm{SD} ; n=34$; Asterisks indicate significant differences between treatments, ${ }^{*}$ significant at $p<0.05 ;{ }^{* *}$ significant at $p<0.01 ;{ }^{* * *}$ significant at $p<0.001$. PWV $(\mathrm{C}-\mathrm{R})$ = carotid-radial pulse-wave pressure; PVW $(\mathrm{C}-\mathrm{F})=$ carotid-femoral pulse-wave pressure; RI = reflection index; SI = stiffness index. ${ }^{b} 50$ - $250 \mathrm{mg}$ acetylsalicylic acid taken on awakening (normal administration); ${ }^{c} 50$ - $250 \mathrm{mg}$ acetylsalicylic acid taken at bedtime for 3 months; ${ }^{d} n=33 ;{ }^{e} n=32$. 
The time of administration did not have statistically significant effect on the average ambulatory blood pressure values nor other parameters measured (Table 2 and Table 3). However, Table 4 shows that the change in the time in the ASA administration from morning to evening had a statistically significant decreasing effect on the relative changes between the diurnal and the nocturnal systolic ambulatory BP. That trend can also be noticed in Figures 1(a) and Figures 1(b).

There was a significant correlation between age and RI, BMI and RI, and BMI and SI (after both the morning and the evening administration of ASA), between sex and carotid-radial PWV (after the morning administration), as well as between age and SI (after the evening administration) (Table 5).

The patients with the carotid-femoral PWV under or equal to $10.0 \mathrm{~m} / \mathrm{s}(\mathrm{n}=15)$ to start with also had statistically significantly lower office SDP (average $131.7 \mathrm{mmHg}$ ) compared with the patients that had the carotid-femoral PWV over $10.0 \mathrm{~m} / \mathrm{s}(\mathrm{n}=19)$ (SDP average $142.8 \mathrm{mmHg}$ ). In both groups, the office SBP decreased after ASA had been taken in the evening for three months. The difference was statistically significant only in the group of patients with higher carotid-femoral PWV.

\section{Discussion}

\subsection{Summary}

Our data shows such a trend that the morning administration of ASA specifically lowers diurnal ambulatory BP, both systolic and diastolic. Similarly, the evening administration of ASA seems to have a lowering effect on the nocturnal systolic and diastolic ambulatory BP. Also, a trend was noticed that the morning administration of ASA seems to have a lowering effect on the systolic BP measured at home in the evening, and the evening administration of ASA on the systolic BP measured at home in the morning. Thus, the results of our study suggest that the lowering effect of ASA on the BP seems to last about $12 \mathrm{~h}$, but not longer. However, this requires further research in a larger group of patients.

\begin{tabular}{|c|c|c|}
\hline Measure & Morning ASA $^{b}$ & Evening ASA $^{c}$ \\
\hline 24 h syst BP (mmHg) & $133.6 \pm 15.6$ & $133.7 \pm 12.3$ \\
\hline $24 \mathrm{~h}$ diast $\mathrm{BP}(\mathrm{mmHg})$ & $77.0 \pm 8.0$ & $77.7 \pm 7.8$ \\
\hline 24 h MAP (mmHg) & $97.6 \pm 9.1$ & $97.9 \pm 8.2$ \\
\hline $24 \mathrm{~h}$ pulse pressure (mmHg) & $56.8 \pm 13.3$ & $57.0 \pm 11.6$ \\
\hline $24 \mathrm{~h}$ heart rate (bpm) & $71.8 \pm 10.9$ & $72.6 \pm 11.4$ \\
\hline 24 h day syst BP (mmHg) & $136.6 \pm 15.8$ & $138.8 \pm 14.2$ \\
\hline 24 h day diast BP (mmHg) & $79.8 \pm 8.3$ & $81.9 \pm 9.3$ \\
\hline 24 h day MAP (mmHg) & $100.4 \pm 9.7$ & $102.5 \pm 9.8$ \\
\hline 24 h day pulse pressure (mmHg) & $57.0 \pm 13.3$ & $57.1 \pm 12.1$ \\
\hline 24 h RR: day heart rate (bpm) & $74.5 \pm 10.5$ & $75.8 \pm 12.4$ \\
\hline 24 h night syst $\mathrm{BP}(\mathrm{mmHg})^{d}$ & $129.2 \pm 16.4$ & $124.9 \pm 12.8$ \\
\hline $24 \mathrm{~h}$ night diast $\mathrm{BP}(\mathrm{mmHg})^{d}$ & $71.7 \pm 8.6$ & $70.3 \pm 7.5$ \\
\hline $24 \mathrm{~h}$ night MAP $(\mathrm{mmHg})^{d}$ & $92.8 \pm 10.2$ & $89.7 \pm 8.1$ \\
\hline $24 \mathrm{~h}$ night pulse pressure $(\mathrm{mmHg})^{d}$ & $57.2 \pm 13.5$ & $54.8 \pm 11.3$ \\
\hline 24 h RR: night heart rate (bpm) ${ }^{d}$ & $66.7 \pm 12.1$ & $66.9 \pm 11.1$ \\
\hline
\end{tabular}

${ }^{a}$ Results expressed as mean $\pm \mathrm{SD} ; n=33$; Asterisks indicate significant differences between treatments, ${ }^{*}$ significant at $\mathrm{p}<0.05 ;{ }^{* *}$ significant at $\mathrm{p}<0.01$; ${ }^{* * * *}$ significant at $p<0.001$. MAP $=$ mean arterial pressure; ${ }^{b} 50$ - $250 \mathrm{mg}$ acetylsalicylic acid taken on awakening (normal administration); ${ }^{c} 50$ - $250 \mathrm{mg}$ acetylsalicylic acid taken at bedtime for 3 months; ${ }^{d} n=32$. 
Table 4. Circadian variation in home and ambulatory $\mathrm{BP}^{a}$.

\begin{tabular}{|c|c|c|}
\hline Measure & Morning ASA $^{b}$ & Evening $\mathrm{ASA}^{c}$ \\
\hline Syst BP morning, home ${ }^{d}$ & $136.7 \pm 18.4$ & $135.8 \pm 16.9$ \\
\hline Syst BP evening, home ${ }^{e}$ & $135.2 \pm 16.6$ & $136.5 \pm 15.4$ \\
\hline Change & $-1.6 \pm 6.8$ & $0.7 \pm 5.8$ \\
\hline Relative change (\%) & $-0.9 \pm 5.0$ & $0.7 \pm 4.3$ \\
\hline Diast BP morning, home ${ }^{d}$ & $81.7 \pm 9.9$ & $81.7 \pm 9.4$ \\
\hline Diast BP evening, home ${ }^{e}$ & $80.1 \pm 10.6$ & $81.2 \pm 9.3$ \\
\hline Change & $-1.6 \pm 4.0$ & $-0.3 \pm 3.1$ \\
\hline Relative change (\%) & $-1.9 \pm 4.7$ & $-0.3 \pm 3.7$ \\
\hline 24 h day syst BP (mmHg) & $136.6 \pm 15.8$ & $138.8 \pm 14.2$ \\
\hline 24 h night syst BP $(\mathrm{mmHg})^{e}$ & $129.2 \pm 16.4$ & $124.9 \pm 12.8$ \\
\hline Change & $-8.1 \pm 7.6$ & $-14.4 \pm 12.3$ \\
\hline Relative change (\%) & $-5.9 \pm 5.7^{* *}$ & $-10.0 \pm 8.1^{* *}$ \\
\hline 24 h day diast BP (mmHg) & $79.8 \pm 8.3$ & $81.9 \pm 9.3$ \\
\hline $24 \mathrm{~h}$ night diast $\mathrm{BP}(\mathrm{mmHg})^{e}$ & $71.7 \pm 8.6$ & $70.3 \pm 7.5$ \\
\hline Change & $-8.2 \pm 6.5$ & $-11.6 \pm 7.7$ \\
\hline Relative change (\%) & $-10.0 \pm 8.2^{*}$ & $-13.7 \pm 8.4^{*}$ \\
\hline
\end{tabular}

${ }^{a}$ Results expressed as mean $\pm \mathrm{SD} ; n=34$; Asterisks indicate significant differences in relative changes between treatments, ${ }^{*}$ significant at $p<0.05 ;{ }^{* *}$ significant at $p<0.01 ;{ }^{* * *}$ significant at $p<0.001 ;{ }^{b} 50-250 \mathrm{mg}$ acetylsalicylic acid taken on awakening (normal administration); ${ }^{c} 50$ $250 \mathrm{mg}$ acetylsalicylic acid taken at bedtime for 3 months; ${ }^{d} n=33 ;{ }^{e} n=32$.

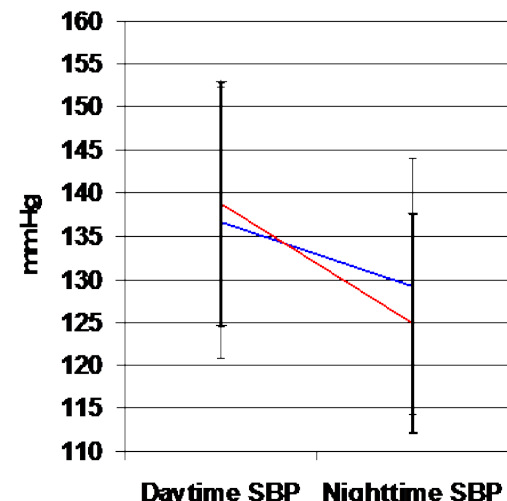

(a)

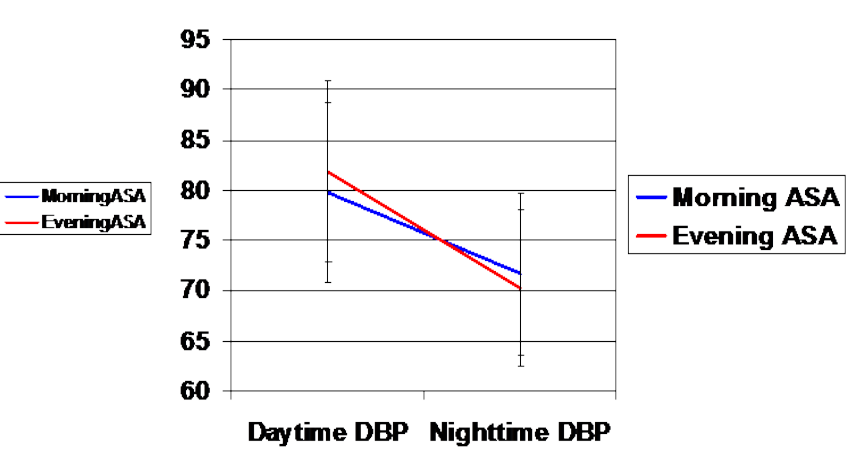

(b)

Figure 1. (a) The daytime and nighttime systolic blood pressure (SBP) after morning or evening administration of acetylsalicylic acid (ASA). (b) The daytime and nighttime diastolic blood pressure (DBP) after morning or evening administration of acetylsalicylic acid (ASA).

\subsection{Limitations of the Study}

The number of the study patients was low and the study set-up non-randomized. This set-up was however straightforward and was easily applied to the patient flow at the outpatient clinic. Further studies with randomization and larger patient material are warranted to test the findings of the present study.

\subsection{Main Findings}

Our study showed a significant decrease in the office SBP after the evening administration of ASA. However, 
Table 5. Correlations between selected variables ${ }^{a}$.

\begin{tabular}{lcccc}
\hline \multirow{2}{*}{ Variable } & \multicolumn{2}{c}{ Morning } & \multicolumn{2}{c}{ Evening } \\
\cline { 2 - 5 } & $r$ & $P$ value & $r$ & $P$ value \\
\hline Sex $^{b}$ - Systolic BP (mmHg) & 0.08 & 0.668 & 0.08 & 0.644 \\
Diastolic BP (mmHg) & 0.21 & 0.225 & -0.07 & 0.681 \\
PWV (C-R) & 0.48 & $0.004^{* *}$ & 0.22 & 0.219 \\
PWV (C-F) & 0.09 & 0.619 & 0.12 & 0.514 \\
RI & -0.07 & 0.706 & 0.01 & 0.959 \\
SI & 0.09 & 0.619 & 0.21 & 0.226 \\
Age - Systolic BP (mmHg) & 0.09 & 0.610 & 0.02 & 0.892 \\
Diastolic BP (mmHg) & -0.21 & 0.243 & -0.18 & 0.304 \\
PWV (C-R) & -0.08 & 0.642 & -0.07 & 0.699 \\
PWV (C-F) & 0.24 & 0.180 & 0.12 & 0.507 \\
RI & 0.44 & $0.009^{* *}$ & 0.52 & $0.001^{* *}$ \\
SI & 0.16 & 0.367 & 0.39 & $0.022^{*}$ \\
BMI - Systolic BP (mmHg) & 0.27 & 0.119 & 0.11 & 0.525 \\
Diastolic BP (mmHg) & 0.26 & 0.142 & 0.33 & 0.054 \\
PWV (C-R) & -0.02 & 0.908 & 0.23 & 0.193 \\
PWV (C-F) & -0.03 & 0.877 & 0.19 & 0.274 \\
RI & -0.34 & $0.047^{*}$ & -0.51 & $0.002^{* *}$ \\
SI & -0.47 & $0.005^{* *}$ & -0.49 & $0.003^{* *}$ \\
\hline
\end{tabular}

${ }^{a} r=$ Pearson's correlation coefficient (continuous variables), Spearmann's rho correlation coefficient (categorical variables). " significant at $p<0.05 ;{ }^{* *}$ significant at $p<0.01$. PWV (C-R) = carotid-radial pulse-wave pressure; PVW $(\mathrm{C}-\mathrm{F})$ = carotid-femoral pulse-wave pressure; $\mathrm{RI}=$ reflection index; $\mathrm{SI}=$ stiffness index. ${ }^{b}$ Sex: $1=$ female; 2 = male.

the decrease was observed only in the office BP measurements and therefore this study does not show a clear difference in the effect of the morning versus evening administration of ASA on the ambulatory or the home blood pressure. The decrease in the office SBP can be a result of familiarization. During the first study visit, the patients could have had higher BP due to nervousness but during the later visit, they were already familiar with the procedure and the investigator. The time of the year can also have an effect on the blood pressure.

Previous studies by Hermida et al. have suggested that the evening administration of ASA decreases the ambulatory SBP and DBP [2]-[7]. All of the studies by Hermida et al. were randomized and some of them [6] [7] were reported to follow the PROBE design (prospective, randomized, open-label, blinded endpoint). The study by Dimitrov et al. [8] followed a randomized cross-over design. In our study, the subjects were studied as one group. Initially ASA was taken in the morning and after the first set of measurements, ASA was taken in the evening.

In our study we noticed a higher difference in the circadian variation of the blood pressure after the evening administration of ASA. Daytime blood pressure was lower after the morning administration of ASA but the evening administration of ASA decreased the nocturnal blood pressure more than the morning ASA administration. Our results suggest that ASA may have a blood pressure decreasing effect that does not last 24 hours. In the study by Dimitrov et al. (2012), significant differences were not found in the diurnal and nocturnal SBP or DBP [8]. Also Bonten et al. (2015) and Lafeber et al. (2014) observed no differences in BP between morning and bed time administration of ASA [9] [10]. To confirm the results of our study larger-scale studies are warranted.

Another result in our study was the higher carotid-femoral PWV after the evening administration of ASA. One explanation for this may be that ASA has some effect on carotid-femoral PWV which manifests when ASA is administered 1 - 2 hours before the measurement, but not 10 - 12 hours before it. The effect of antihyperten- 
sive medication cannot explain the phenomenon because the patients took antihypertensive medication in both measurements in the morning. Blood pressure was lower in the patients with lower carotid-femoral PWV which has also been seen in earlier studies [16].

The significant increase in plasma triglycerides could possibly be explained by the time of the year the study was conducted. The first set of measurements was done in the spring and the second set in the autumn. During the summer the diet usually changes, which could explain the rise.

\subsection{Conclusions}

As a conclusion, ASA administration seems to lower BP for less than 24 hours. Therefore, the time of the ASA administration seems to affect the circadian variation in the BP. Other clinically significant effects were not observed.

\section{Acknowledgements}

The authors thank Jukka-Pekka Suomela for his statistical assistance. This study was financially supported by Turku University Hospital.

\section{Declaration}

None of the Authors has financial or other relationships that might lead to a conflict of interest. The manuscript has been read and approved by all authors. The study is academically funded.

\section{References}

[1] de Gaetano, G. (2001) Collaborative Group of the Primary Prevention Project. Low-Dose Aspirin and Vitamin E in People at Cardiovascular Risk: A Randomized Trial in General Practice. Lancet, 357, 89-95. http://dx.doi.org/10.1016/S0140-6736(00)03539-X

[2] Hermida, R.C., Ayala, D.E., Fernández, J.R., Mojón, A., Alonso, I., Silva, I., et al. (1999) Administration Time-Dependent Effects of Aspirin in Women at Differing Risk for Pre-Eclampsia. Hypertension, 34, 1016-1023. http://dx.doi.org/10.1161/01.HYP.34.4.1016

[3] Hermida, R.C., Ayala, D.E., Iglesias, M. and Halberg, F. (1994) Time-Dependent Effects of ASA Administration on Blood Pressure in Healthy Subjects. Chronobiologia, 21, 201-213.

[4] Hermida, R.C., Mojón, A. and Fernandez, J.R. (2009) Ambulatory Blood Pressure Control with Bedtime Administration in Subjects with Prehypertension. American Journal of Hypertension, 22, 896-903. http://dx.doi.org/10.1038/ajh.2009.83

[5] Hermida, R.C., Calvo, C., Ayala, D.E., Dóminguez, M.J., Covelo, M., Férnandez, J.R., et al. (2003) Administration Time-Dependent Effects of Aspirin on Blood Pressure in Untreated Hypertensive Patients. Hypertension, 41, 12591267. http://dx.doi.org/10.1161/01.HYP.0000072335.73748.0D

[6] Hermida, R.C., Calvo, C. and Lopez, J.E. (2005) Aspirin Administered at Bedtime, But Not on Awakening, Has an Effect on Ambulatory Blood Pressure in Hypertensive Patients. Journal of the American College of Cardiology, 46, 975-983. http://dx.doi.org/10.1016/j.jacc.2004.08.071

[7] Hermida, R.C., Calvo, C., Ayala, J.E., Mojón, A., Rodriguez, M., Chayán, L., et al. (2005) Differing Administration Time-Dependent Effects of Aspirin on Blood Pressure in Dipper and non-Dipper Hypertensives. Hypertension, 46, 1060-1068. http://dx.doi.org/10.1161/01.HYP.0000172623.36098.4e

[8] Dimitrov, Y., Baguet, J.-P., Hottelart, C., Marboeuf, P., Tartiere, J.-M., Ducher, M. and Fauvel, J.-P. (2012) Is There a BP Benefit of Changing the Time of Aspirin Administration in Treated Hypertensive Patients? European Journal of Cardiovascular Prevention \& Rehabilitation, 19, 706-711. http://dx.doi.org/10.1177/1741826711418165

[9] Bonten, T.N., Snoep, J.D., Assendelft, W., Zwaginga, J.J., Eikenboom, J., Huisman, M.V., et al. (2015) Time-Dependent Effects of Aspirin on Blood Pressure and Morning Platelet Activity. A Randomized Cross-Over Trial. Hypertension.

[10] Lafeber, M., Grobbee, D.E., Schrover, I.M., Thom, S., Webster, R., et al. (2014) Comparison of a Morning Polylpill, Evening Polypill and Insividual Pills on LDL-Cholesterol, Ambulatory Blood Pressure and Adherence in High-Risk Patients; a Randomized Cross-Over Trial. International Journal of Cardiology, 181C, 193-199.

[11] Blacher, J., Asmar, R., Djane, S., London, G.M. and Safar, M.E. (1999) Aortic Pulse Wave Velocity as a Marker of 
Car-diovascular Risk in Hypertensive Patients. Hypertension, 33, 1111-1117. http://dx.doi.org/10.1161/01.HYP.33.5.1111

[12] Millasseau, S.C., Ritter, J.M., Takazawa, K. and Chowienczyk, P.J. (2006) Contour Analysis of the Photoplethysmographic Pulse Measured at the Finger. Journal of Hypertension, 24, 1449-1456. http://dx.doi.org/10.1097/01.hjh.0000239277.05068.87

[13] Chowienczyk, P.J., Kelly, R.P., MacCallum, H., Millasseau, S.C., Andersson, T.L., Gosling, R.G., Ritter, J.M. and Anggard, E.E. (1999) Photoplethysmographic Assessment of Pulse Wave Reflection: Blunted Response to Endothelium-Dependent $\beta 2$-Adrenergic Vasodilation in Type II Diabetes Mellitus. Journal of the American College of Cardiology, 34, 2007-2014. http://dx.doi.org/10.1016/S0735-1097(99)00441-6

[14] Sollinger, D., Mohaupt, M.G., Wilhelm, A., Uehlinger, D., Frey, F.J. and Eisenberger, U. (2006) Arterial Stiffness Assessed by Digital Volume Pulse Correlates with Comorbidity in Patients with ESRD. American Journal of Kidney Diseases, 48, 456-463. http://dx.doi.org/10.1053/j.ajkd.2006.05.014

[15] Woodman, R.J., Kingwell, B.A., Beilin, L.J., Hamilton, S.E., Dart, A.M. and Watts, G.F. (2005) Assessment of Central and Peripheral Arterial Stiffness: Studies Indicating the Need to Use a Combination of Techniques. American Journal of Hypertension, 18, 249-260. http://dx.doi.org/10.1016/j.amjhyper.2004.08.038

[16] Parati, G., Stergiou, G.S., Asmar, R., Bilo, G., deLeeuw, P., Imai, Y., et al. (2008) European Society of Hypertension Guidelines for Blood Pressure Monitoring at Home: A Summary Report of the Second International Consensus Conference on Home Blood Pressure Monitoring. Journal of Hypertension, 26, 1505-1530. http://dx.doi.org/10.1097/HJH.0b013e328308da66 